\title{
Chromosomes of the coral Goniopora lobata (Anthozoa: Scleractinia)
}

\section{A. J. Heyward}

Department of Marine Biology, James Cook University of North Queensland, Qld., 4811, Australia.

This study presents the first karyotype of a scleractinian coral. Goniopora lobata is a dioecious, tropical coral whose larvae develop externally after the synchronous release of eggs and sperm. In the first $\mathbf{1 2}$ hours of development the embryos are an excellent source of metaphase material and allow good chromosome preparations to be made. The karyotype of $28(n=14)$ chromosomes, between 1 and 5 microns long, consists of nine metacentric and five submetacentric pairs. A subtelocentric secondary constriction is present on one pair of metacentrics.

\section{INTRODUCTION}

There is very little information of any kind about anthozoan cytogenetics. In the sole study of scleractinian coral chromosomes (Wijsman and Wijsman-Best, 1973), the use of somatic tissue from adult colonies to establish mitotic figures was only partially successful. No exact chromosome counts were established. Subsequently, no further work on coral chromosomes has been reported.

Recently, it has become apparent that the majority of coral species reproduce by releasing gametes into the water, followed by external fertilisation and development (Harrison et al., 1984). In the present study the rapidly dividing cells of such externally developing coral embryos have been utilised to provide the first chromosome preparations from a tropical reef coral.

\section{MATERIALS AND METHODS}

Field work was done at the Orpheus Island Research Station, $70 \mathrm{~km}$ northeast of Townsville, Australia. Five colonies of the dioecious coral $G$. lobata were collected from the fringing reef adjacent to the research laboratory, two days prior to the predictable spawning event (Harrison et al., 1984). Colonies were held in aquaria until they spawned. The released gametes were mixed together and transferred to jars of seawater for culture. Continuous agitation of the developing embryos was essential. Approximately 9 hours after spawning, embryogenesis had proceeded to
128- 256 cell stages. The embryos were then treated with either $0.02,0.05$ or 0.1 per cent $(\mathrm{w} / \mathrm{v})$ colchicine-seawater for 2 hours with continual agitation. They were subsequently rinsed once in clean seawater and immediately fixed in cold $\left(4^{\circ} \mathrm{C}\right)$ glacial acetic acid-absolute ethanol $(1 / 3, \mathrm{v} / \mathrm{v})$. Fixed material was stored at $4^{\circ} \mathrm{C}$.

\section{Squash preparation}

Prior to staining the fixed embryos were soaked in ethyl ether for 2 hours. This removed most of the lipid reserves in the cells which otherwise made light microscopy difficult. Embryos were then placed in a drop of lacto-acetic orcein on a coverslip for 15 minutes prior to the application of a glass slide and firm squashing. Preparations were photographed using transmitted green light and a $100 \times$ oil immersion objective (Olympus BHS 312 ). Micrographs were recorded on Kodak technical pan 2415 film developed for maximum contrast.

\section{RESULTS}

Each colchicine treatment yielded material of similar appearance. In some embryos very large numbers of metaphase stages were seen. The chromosomes were often highly contracted. When there was good separation such plates were used for counts. A survey of 50 plates identified a complement of 28 chromosomes. However, it was not uncommon to find individual chromosomes widely dispersed during squashing, resulting in incomplete nuclei. In some preparations such 


\section{il}


II
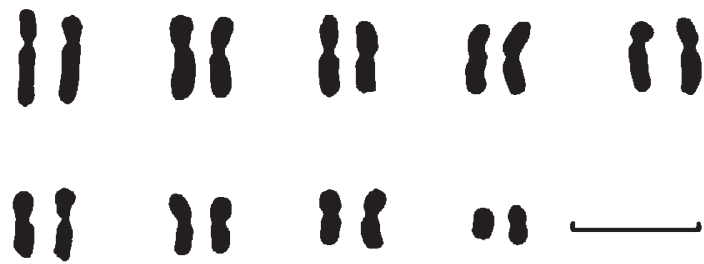

Figure 1 Goniopora lobata. Arrangement of a typical chromosome complement. Arrows indicate the secondary constriction on pair 5 . Scale bar equals $5 \mu \mathrm{m}$.

disassociated chromosomes formed large aggregations of stained material.

The rarer preparations containing elongated chromosomes enabled both counts and ploidy to be determined. Ten such plates revealed that the complement of 28 chromosomes contained unique pairs, presumably reflecting the diploid nature of the karyotype $(n=14)$. The elongated chromosomes ranged in length from 1-5 $\mu \mathrm{m}$. In most cases the centromere was obvious. The karyotype has been arranged according to chromosome length and centromere position (fig. 1). The means and
Table 1 Goniopora lobata. Relative lengths and centromeric indices of the fourteen chromosome pairs; means and standard deviations obtained from the measurements of five metaphase plates

\begin{tabular}{rrr}
\hline Chromosome & Relative length & Centromeric index \\
\hline 1 & $100 \cdot 0 \pm 4 \cdot 8$ & $32 \cdot 5 \pm 1 \cdot 3$ \\
2 & $83 \cdot 6 \pm 1 \cdot 5$ & $33 \cdot 2 \pm 2 \cdot 2$ \\
3 & $80 \cdot 2 \pm 5 \cdot 1$ & $36 \cdot 1 \pm 4 \cdot 6$ \\
4 & $76 \cdot 9 \pm 3 \cdot 6$ & $43 \cdot 4 \pm 3 \cdot 8$ \\
5 & $75 \cdot 1 \pm 4 \cdot 6$ & $40 \cdot 3 \pm 3 \cdot 6$ \\
6 & $69 \cdot 3 \pm 3 \cdot 1$ & $37 \cdot 3 \pm 3 \cdot 0$ \\
7 & $63 \cdot 0 \pm 2 \cdot 0$ & $40 \cdot 9 \pm 2 \cdot 7$ \\
8 & $60 \cdot 2 \pm 4 \cdot 7$ & $42 \cdot 9 \pm 2 \cdot 6$ \\
9 & $54 \cdot 4 \pm 3 \cdot 4$ & $45 \cdot 0 \pm 5 \cdot 2$ \\
10 & $53 \cdot 7 \pm 5 \cdot 5$ & $37 \cdot 3 \pm 3 \cdot 6$ \\
11 & $49 \cdot 8 \pm 2 \cdot 2$ & $41 \cdot 7+2 \cdot 5$ \\
12 & $49 \cdot 3 \pm 3 \cdot 7$ & $45 \cdot 6 \pm 3 \cdot 5$ \\
13 & $43 \cdot 1 \pm 4 \cdot 7$ & $41 \cdot 8 \pm 1 \cdot 8$ \\
14 & $32 \cdot 8 \pm 3 \cdot 5$ & $50 \cdot 0 \pm 2 \cdot 7$ \\
\hline
\end{tabular}

standard deviations of the relative lengths and centromeric indices of the fourteen chromosome pairs are given in table 1 . The idiogram (fig. 2) is derived from these values. According to the nomenclature of Levan et al. (1964) there are nine metacentric and five submetacentric pairs. Most pairs are close to metacentric. There is a steady decrease in size from pair $1-13$, then a more pronounced step down from pair 13-14. One poten-

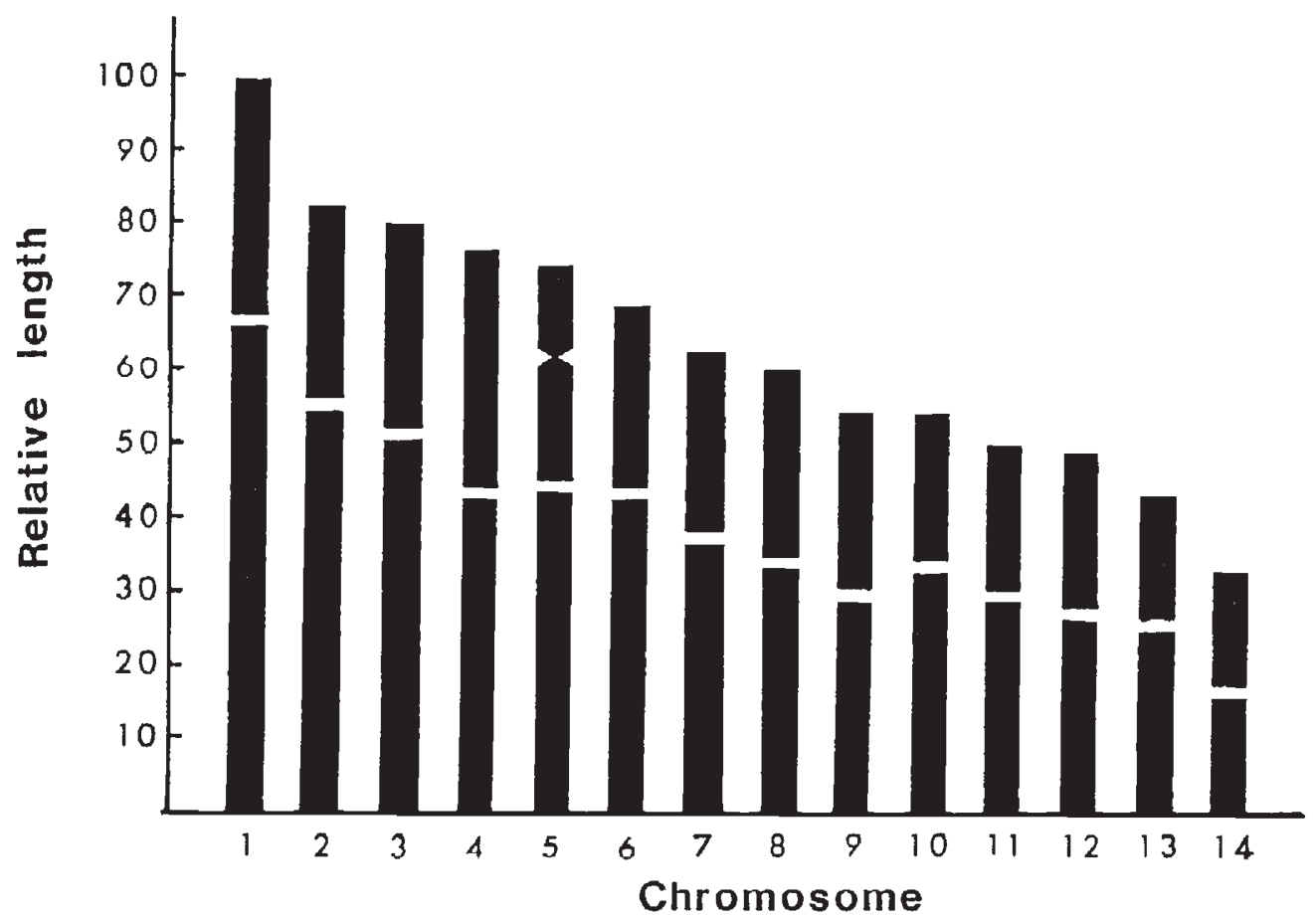

Figure 2 Goniopora lobata. Idiogram of one set of diploid chromosomes $(2 n=28)$. 
tially useful marker of the karyotype is the secondary subtelocentric constriction present on pair 5 (figs. 1 and 2). However this constriction was not always obvious.

\section{DISCUSSION}

These are the first quantitative data on coral chromosomes. The majority of coral species have external development (Babcock et al., unpubl. data) and consequently lend themselves to the karyological techniques employed here. Accurate prediction of gamete spawning is now possible for species in Australia (Harrison et al., 1984), the Red Sea (Loya, pers. comm.) and Hawaii (Krupp, 1983). Hence a large number of species over a wide geographic range are now accessible for cytogenetic investigation. A complement of 28 chromosomes between one and five microns long represents an encouraging karyotype for further work. As the methods used here are improved, studies are continuing on the application of karyotypic data to anthozoan systematics. Preliminary observations (author, unpubl. data) sug- gest that chromosome number may be highly conservative among the Scleractinia.

Acknowledgements I wish to especially thank L. Winsor and Z. Florian for their instruction, encouragement and generous assistance in preparing and photographing the material. Prof. B. John and Dr B. Jackes provided much appreciated advise and assistance with interpretation. This study was supported by an Australian Postgraduate Research Award and the Scleractinian Coral Research Unit at James Cook University.

\section{REFERENCES}

HARRISON, P. G., BABCOCK, R., BULL, G., OLIVER, J. WAllaCE, C. AND W11_Lis, B. 1984. Mass Spawning in tropical reef corals. Science, 223, 1186-1189.

KRUPP, D. A. 1983. Sexual reproduction and early development of the solitary coral Fungia scutaria (Anthozoa: Scleractinia). Coral Reefs, 2, 159-164.

LEVAN, A., FREDGA, K. AND SANDBERG, A. A. 1964. Nomenclature for centromeric position on chromosomes. Hereditas, 52, 201-220.

WIJSMAN, H. J. AND WIJSMAN-BEST, M. 1973. A note on the chromosomes of some madreporarian corals from the Mediterranean (Cnidaria, Anthozoa, Scleractinia: Dendrophyllidae, Caryophyllidae, Faviidae). Genen Phenen, 16(2), 61-64. 